\title{
MONTE CARLO SIMULATION OF HEISENBERG SPIN GLASS ON FCC LATTICE IN EXTERNAL MAGNETIC FIELD
}

\author{
J. MLODZKI a , F.R. WUENSCH ${ }^{b}$ and R.R. GALĄZKA ${ }^{a}$ \\ anstitute of Physics, Polish Academy of Science, Al. Lotników 32/46, 02-668 Warsaw, Poland \\ ${ }^{b}$ Department of Physics, University of Regensburg, D 8400 Regensburg. Fed. Rep. Germany
}

Received 11 July 1989; in revised form 16 October 1989

\begin{abstract}
The simple but realistic model described by a Heisenberg Hamiltonian with nearest neighbours and next nearest neighbours interactions in an external magnetic field was investigated by use of the Monte Carlo method. Three-dimensional vector spins of length $\frac{5}{2}$ were distributed randomly on a fcc lattice. Different concentrations of spins, $x=0.05,0.10, \ldots, 0.90$, were studied. For low concentrations, simulated samples contained about 1000 spins. For higher values of $x$ the size of system was about 8000. All the computations were done for high external magnetic fields of around $3 \mathrm{~T}$. During simulation, physical quantities such as magnetization, energy, specific heat and magnetic susceptibility were determined. The results for magnetization differ for the zero-field-cooled (ZFC) and field-cooled (FC) cases for the whole range of concentrations. This difference, also typical for experimental data, seems to vanish after longer simulation. From critical temperatures for computer simulated magnetization, the magnetic phase diagram was obtained and compared to experimental data for $\mathrm{Cd}_{1-x} \mathbf{M n}_{x} \mathrm{Te}$. Concentration dependent results for magnetization, specific heat and magnetic susceptibility allowed one to distinguish three different regions for the simulated system: $x \leq 0.20,0.30 \leq x \leq 0.60,0.70 \leq x \leq 0.90$.
\end{abstract}

\section{Introduction}

Semimagnetic semiconductors (SMSC) or diluted magnetic semiconductors (DMS) are mixed crystals or solid solutions of semiconductor compounds, such as eg. $\mathrm{CdTe}, \mathrm{HgSe}$ and $\mathrm{PbS}$, with appropriate magnetic compounds like MnTe, MnSe or MnS [1].

$\mathrm{Cd}_{1-x} \mathrm{Mn}_{x} \mathrm{Te}$, a member of this group of materials, crystallizes in zinc-blende structure over the whole $x \leq 0.70$ range [2]. The $\mathrm{Cd}^{2+}$ and $\mathrm{Mn}^{2+}$ ions randomly populate a foc sublattice. Manganese ions are mainly coupled by the nearest neighbour (NN) antiferromagnetic (AF) superexchange interaction. The next nearest neighbour (NNN) and more distant interactions are weaker and decrease very rapidly with distance between the $\mathrm{Mn}$ ions [3]. The value of $J_{\mathrm{NNN}}$ is not well known and is believed to be in range $0.1-0.25$ of the NN exchange constant [4-6].
The fcc lattice with AF couplings does not allow to simultaneous minimization of energies of all exchange bonds and thus leads to frustration. Randomness and frustration are two essential factors thought to be responsible for the spin-glass behaviour observed experimentally in SMSC [7].

In our paper we present a simple computer model of such materials and compare Monte Carlo simulation results with available experimental data. Such an approach was used by Kett, Gebhardt and Krey [8] to investigate magnetization behaviour of $\mathrm{Cd}_{0.45} \mathrm{Mn}_{0.55} \mathrm{Te}$ mixed crystals in high external magnetic fields. We extended use of this model for the whole range of concentrations of Mn ions (including the physically inaccessible values of $x=0.80,0.90)$ and determined also the energy, magnetic susceptibility and specific heat.

In section 2 the details of the model, definitions of observed physical quantities. and procedures of 
the Monte Carlo simulations are described. Section 3 contains our results and comparison with experiment. In section 4 we present final remarks and conclusions.

\section{Physical model and conditions of the Monte Carlo simulation}

\subsection{Model for simulation}

In the present model spins are distributed randomly over an foc lattice with imposed cyclic boundary conditions. The number of fcc sites is $N_{\mathrm{g}}$ and the number of spins, $N_{\mathrm{s}}$, and these are related to the concentrations of spins, $x$, by the formula

$x=N_{\mathrm{s}} / N_{\mathrm{g}}$.

Each spin is coupled by exchange interactions with its nearest neighbours (up to 12) and with its next nearest neighbours (up to 6). Spins also interact with the external magnetic field.

The total interaction is given by the following Heisenberg Hamiltonian

$H=-J_{\mathrm{nn}} \sum S_{i} \cdot S_{j}-J_{\mathrm{nnn}} \sum S_{i} \cdot S_{j}-\frac{g \mu_{\mathrm{B}}}{k} B \cdot \sum S_{i}$.

The first sum is over the pairs of the nearest neighbours, the second over the pairs of next nearest neighbours and the last one over all spins. $J_{n n}$ and $J_{n n n}$ are the respective exchange integrals expressed in kelvin. The external magnetic field $B$ in tesla is applied along the $z$-direction. $\mu_{B}$ is the Bohr magneton, $k$ the Boltzmann constant and $g$ is the Landé $g$-factor.

Here we are concerned only with the classical limit $S \rightarrow \infty$, where the quantum nature of the spins is ignored and the Hamiltonian operator reduces to the internal energy $E$. Spins $S_{i}$ are regarded as three-dimensional vectors

$S_{i}=\left(S_{i}^{x}, S_{i}^{y}, S_{i}^{z}\right)$ with fixed length $S$.

To make computations easier we introduce dimensionless quantities and unit vector spin. It enables us to evaluate the energy by the following formula:

$e=-A_{1} \sum s_{i} \cdot s_{j}-A_{2} \sum s_{i} \cdot s_{j}-b \cdot \sum s_{i}$,

where

$A_{1}=J_{\mathrm{nn}} /\left|J_{\mathrm{nn}}\right|, \quad A_{2}=J_{\mathrm{nnn}} /\left|J_{\mathrm{nn}}\right|$,

$\boldsymbol{b}=\frac{g \mu_{\mathrm{B}}}{k} \boldsymbol{B} /\left(S\left|J_{\mathrm{nn}}\right|\right)$,

$e=E /\left(S^{2}\left|J_{\mathrm{nn}}\right|\right), \quad t=T /\left(S^{2}\left|J_{\mathrm{nn}}\right|\right)$.

and $b, e$ and $t$ are the magnetic field, energy and temperature in dimensionless units. The magnitude of the vector $s_{i}$ is equal to one.

To compare our numerical results with experimental data for $\mathrm{Cd}_{1-x} \mathrm{Mn}_{x} \mathrm{Te}$ we have chosen: $S=\frac{5}{2}, J_{\mathrm{nn}}=-10 \mathrm{~K}$ and $J_{\mathrm{nnn}}=1.25 \mathrm{~K}$ [8]. The concentration of spins in the computer simulation corresponds to the concentration of $\mathrm{Mn}$ ions in the experiment. In general, the same model with different $J_{\text {nn }}$ and $J_{\text {nnn }}$ can be used to simulate other materials containing $\mathrm{Mn}$ ions of spin momentum $S=\frac{5}{2}\left(\mathrm{Hg}_{1-x} \mathrm{Mn}_{x} \mathrm{Te}, \mathrm{Zn}_{1-x} \mathrm{Mn}_{x} \mathrm{Te}\right.$, etc.).

\subsection{Procedures of Monte Carlo}

Our Monte Carlo calculations follow the procedure described by Binder [9]. The basic idea is to generate a representative ensemble of states to be used for the determination of thermodynamical quantities. At the beginning the directions of spins are chosen randomly with a uniform distribution of the spins on the unit sphere. The representative ensemble of states is obtained by rotating the spins one at a time. Each Monte Carlo step (MCS) is defined as a selection of one new configuration of the system by attempting to rotate $N_{\mathrm{s}}$ spins in a random way and with a random selection of sites. Each single rotation is performed following the rule *

$s_{i}^{\alpha}=R(0,1)-0.5$,

\footnotetext{
- All vectors with lengths greater than unity before normalization, are discarded in order to obtain homogeneous distribution of spin vectors in a sphere, not in a cube [9].
} 
with a subsequent rescaling of the spin length. $R(0,1)$ is a random number taken uniformly between 0 and 1. The new direction of the spin is accepted if it leads to a lowering of the total energy of the system. If the energy increases, the factor $\exp (-\Delta e / t)$ is compared against a number chosen randomly and uniformly in the interval from 0 to 1 . If the factor in question is greater than the random number then we accept the new direction of the spin, otherwise it is rejected and the previous one kept. $\Delta e$ is the change in the energy produced by the attempted rotation.

For each configuration of spins, $\boldsymbol{x}_{j}=$ $\left(s_{1}, s_{2}, \ldots, s_{N_{s}}\right)$, obtained after one Monte Carlo step, we determine the magnetization for the average spin

$M\left(x_{j}\right)=\frac{1}{N_{s}} \sum_{i=1}^{N_{s}} s_{i}$,

and the internal energy per spin:

$E\left(x_{j}\right)=\frac{S^{2}}{N_{s}} e\left(x_{j}\right)$,

where $e\left(x_{j}\right)$ is given by eq. (3). The Monte Carlo algorithm described above allows us to reduce ensemble averages to simple arithmetic averages

$$
\begin{aligned}
& \langle M\rangle=\frac{1}{m} \sum_{j=1}^{m} M\left(x_{j}\right), \\
& \langle E\rangle=\frac{1}{m} \sum_{j=1}^{m} E\left(x_{j}\right),
\end{aligned}
$$

where $m$ is the number of configurations which is taken into account. Fluctuations of the energy and magnetization vector enable us to determine the specific heat, $C$ and the tensor of magnetic susceptibility, $\chi$

$$
\begin{aligned}
C= & \frac{N_{\mathrm{s}}}{m T^{2}} \sum_{j=1}^{m}\left(E\left(x_{j}\right)-\langle E\rangle\right)^{2}, \\
\chi_{\alpha \beta}= & \frac{N_{s}}{m T} \sum_{j=1}^{m}\left(M_{\alpha}\left(x_{j}\right)-\left\langle M_{\alpha}\right\rangle\right) \\
& \times\left(M_{\beta}\left(x_{j}\right)-\left\langle M_{\beta}\right\rangle\right),
\end{aligned}
$$

where $\alpha, \beta$ denote Cartesian components.

During simulation, the scatter of specific heat and susceptibility values was greater than for the magnetization and energy. Because of this we also establish $C$ as an energy derivative

$C=\partial E / \partial T$.

For each temperature some number of the Monte Carlo steps should be rejected as not being characteristic of true equilibrium. This number depends on temperature and was automatically chosen by the program in the range 200-1400 MCS. At each temperature, 1000 configurations were taken into account when calculating physical quantities. Then we changed temperature quasicontinuously during $500 \mathrm{MCS}$. To get temperature dependence of the previously mentioned physical quantities we repeated this procedure for different temperatures.

To reproduce experimental measurements of the zero field-cooled system and the field-cooled one we started from a random configuration in the high temperature region $(100 \mathrm{~K})$ and quasicontinuously, during 4000 MCS, cooled down the system (usually to $1 \mathrm{~K}$ ) without the magnetic field. Then, during 500 cycles we increased slowly the value of the field to $2.8 \mathrm{~T}$. After having the field switched-on, we rejected 6000 configurations and then we did the first "measurement". We call this procedure the ZFC landing, because it establishes the starting point for the $\mathrm{ZFC}$ curves.

ZFC simulation continued for the next temperatures by heating the system a little, rejecting some configurations and taking 1000 steps before "measurement". When the system was heated to a high temperature we simulated FC behaviour by cooling the system down slowly and repeating rejection and measurement for each temperature.

\subsection{Various concentrations, number of spins and temperature ranges}

In our numerical experiments we studied different numbers of concentrations of spins, $x$ ranging from 0.05 to 0.90 . For small concentrations up to 0.30 , it was sufficient to take into account relatively small systems containing approximately 1000 spins. With higher concentrations it was rather difficult to get good results - smooth curves for magnetization, specific heat, etc. - even for 
systems of about 8000 spins. We used the following sizes of sample for concentration: $0.40,0.70$, $0.80,0.90$. For 0.50 and 0.60 we did simulation with a rather small system of about 1000 spins. Scatter of the data in these cases is relatively high. We found from our computations that the critical temperature for the $z$-component of magnetization (corresponding to the maximum of ZFC curve) depends on concentration. To investigate this behaviour it was necessary to do experiments in the proper temperature range with respect to the value of the critical temperature for a given concentration. We experimentally found these ranges and the physical results are presented in section 3 .

\section{Results}

\subsection{Temperature-dependent magnetization}

In this section we present results for the ZFC and FC magnetization as a function of temperature and concentration. In fig. $1 \mathrm{a}, \mathrm{b}, \mathrm{c}$ the $z$-components of magnetization are plotted for both cases. Each pair of curves represents a different concentration, $x$ ranging from 0.05 to $0.80 . M_{z}$ is measured relative to its maximum $M_{0}=1$. Temperature is in kelvin and the value of the magnetic field along the $z$-axis is $2.8 \mathrm{~T}$. All our results are consistent with the observation that the FC and ZFC magnetization differs at all $x$, the latter one always being lower. The ZFC magnetization has a maximum which shifts to higher temperature with increasing concentration. Its value also goes down and curves for both $\mathrm{ZFC}$ and FC become flatter for higher $x$. Finding the position of the maximum, marked as an arrow in the plot, is somewhat arbitrary because of the data scatter, but this does not affect the major features. In the experiment $T_{\max }$ is referred to as the freezing (critical) temperature, $T_{\mathrm{F}}$, and is suggested to characterize the transition point between the paramagnetic and spin-glass states [10-14].

In fig. 2 we plotted the magnetic phase diagram for $T_{\max }$ versus concentration. Our numerical results are compared with experimental data from magnetization and susceptibility measurements for $\mathrm{Cd}_{1-x} \mathrm{Mn}_{x} \mathrm{Te}$ without applied field [10-13]. The coincidence of numerical and experimental data is good - which shows that our Monte Carlo magnetization can reproduce the experimental magnetic phase diagram.

The $T_{\max }$ values derived from numerically calculated $M_{z}$ curves are scaled by $J_{\mathrm{nn}}$ according to the formula

$T=t S^{2}\left|J_{\mathrm{nn}}\right|$.

In our calculation we have taken $J_{\mathrm{nn}}=-10 \mathrm{~K}$ [8], but the recently reported value of $J_{\mathrm{nn}}=-13.8$ [15] gives a better fit to the experimental data, especially for concentration of spins, $x$, greater than 0.20 .

\subsection{Temperature-dependent energy and specific heat}

In fig. 3 we present curves for the ZFC energy per spin as a function of temperature for the whole range of concentration. $x=0.05,0.10,0.20$, $0.30,0.40,0.50,0.60,0.70,0.80,0.90$. Samples with higher concentrations have lower energy, but the shapes of the curves are similiar.

In fig. 4 energies are plotted for ZFC and FC systems in the low-temperature range. The separation between the curves is independent of concentration but the point they join together is shifted to higher temperature for higher concentrations. These results are worth stressing, because we calculated the specific heat as a derivative of the energy with respect to the temperature, and the behaviour of the energy curve is reflected in the behaviour of its derivative.

In fig. $5 a, b, c$ the results for the specific heat per spin, $C$, as a function of temperature for different concentrations, $x=0.10,0.40,0.70$ are plotted. The ZFC curve is always below the FC for low temperature and both curves meet at the temperature where the ZFC curve has a maximum. The position of the maximum and its width change with concentration. For low values of $x$ it is rather narrow and becomes wider with higher concentrations, but at $x=0.80$ starts to be sharp again. Also, a shift of the maximum to higher temperature with increasing $x$ is a general tendency. When we compare the results for specific heat and magnetization, we found that below $70 \%$ 

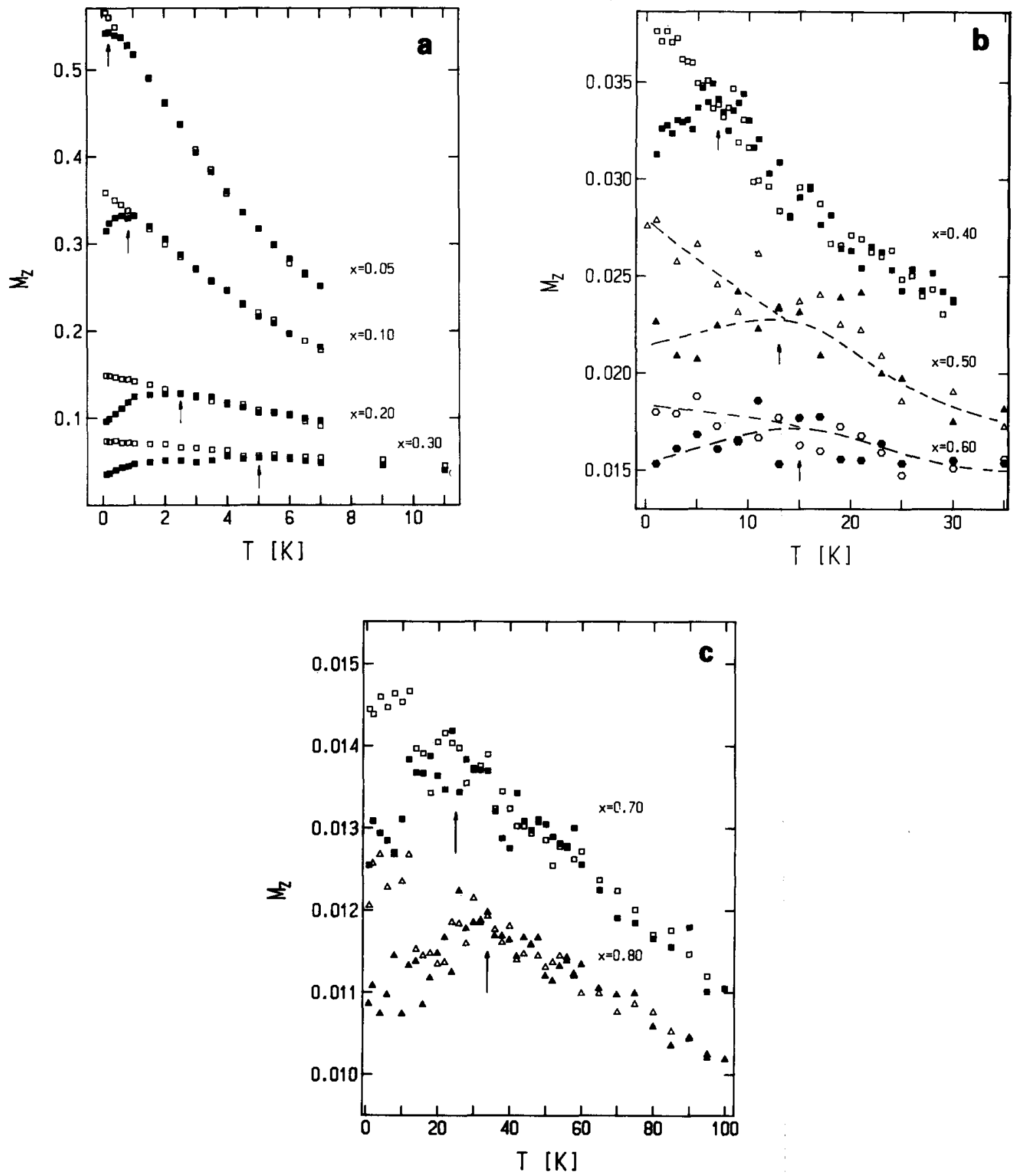

Fig. 1. Temperature dependence of field-cooled (FC) and zero-field-cooled (ZFC) magnetizations for various concentrations of spins, $x$. At low temperatures, for each concentration, the ZFC curve (closed points) is always below the FC one (open points). (a) $x=0.05$, $0.10,0.20,0.30$; (b) $x=0.40,0.50,0.60$; (c) $x=0.70,0.80$. Arrows indicate the critical temperatures. Notice the different temperature ranges and values of magnetization for (a), (b), (c). The dashed line in (b) is a guide for the eye only. 


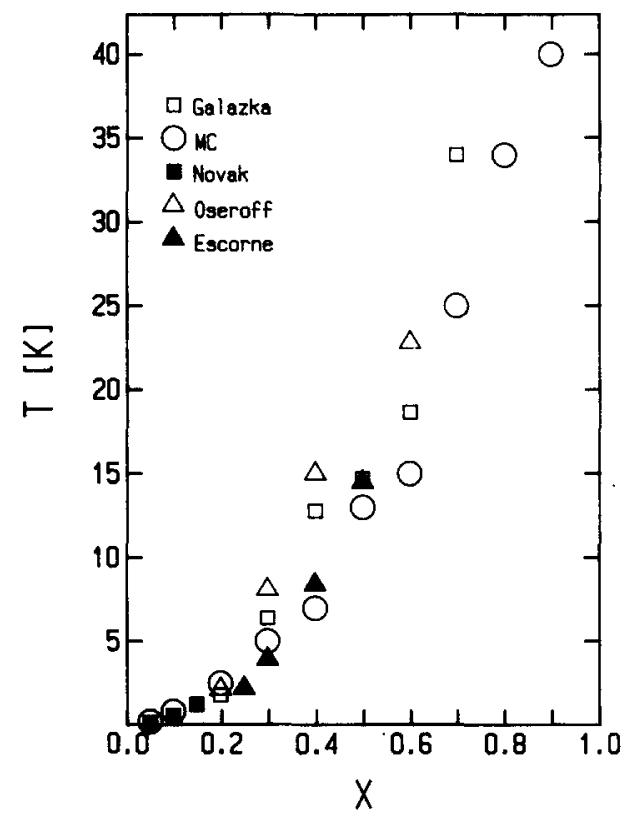

Fig. 2. Magnetic phase diagram of temperature versus concentration of spins, $x$. A comparison is given between the experimental data for $\mathrm{Cd}_{1-x} \mathrm{Mn}_{x} \mathrm{Te}$ [10-13] and our numerical results. $\square$ - ref. [10], $\boldsymbol{\omega}$ - ref. [11], $\Delta$ - ref. [12], $\Delta$ - ref. [13], 0 - our data.

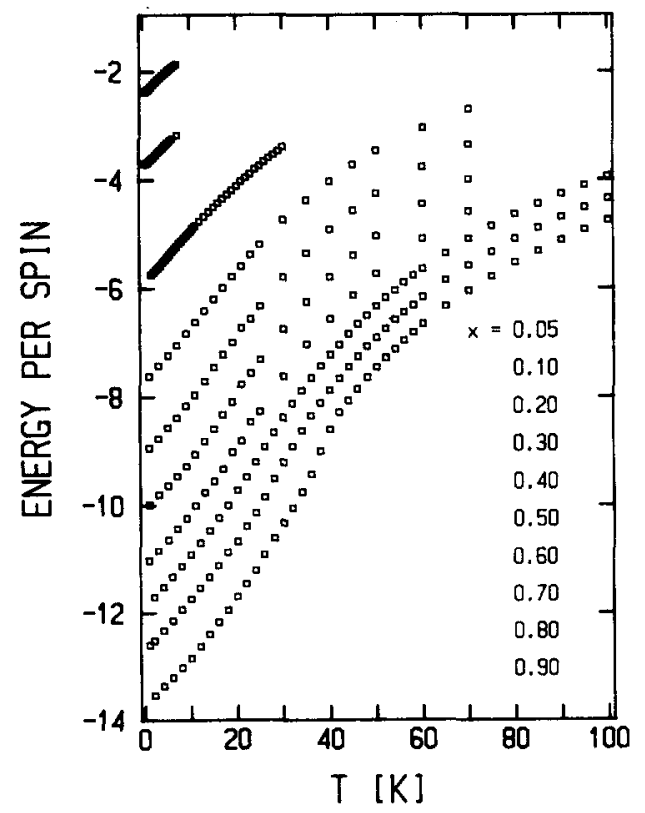

Fig. 3. ZFC energy per spin as a function of temperature for various concentrations of spins, $x=0.05,0.10, \ldots, 0.90$ (from top to bottom).

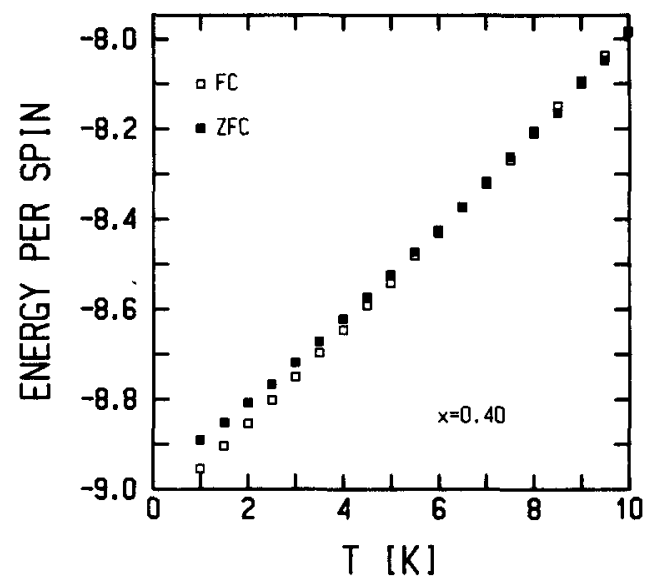

Fig. 4. Energies per spin for ZFC and FC cases in the low temperature range for $x=0.40$.

spin concentration, the maximum for $C$ is at a higher temperature and broader than the respective one for $M_{z}$. At 0.70 and 0.80 the position and width of $M_{z}$ and $C$ extrema are the same.

It must be mentioned that all our results are computed for high external magnetic fields and some difficulties may arise when we try to compare numerical results with experimental measurements done without fields. This is a problem especially for specific heat of low concentration samples where a strong dependence on field is observed [16]. We tested by Monte Carlo simulation the systems with $x=0.30$ for different values of magnetic field. In fig. 6 three curves are plotted for $B=2.8,5.6,8.4 \mathrm{~T}$. Because of little dependence on field for this case, we feel justified to compare our results with experimental data for concentrations starting from $\mathbf{0 . 3 0}$.

It was reported that, in the vicinity of the magnetization cusp, the specific heat behaves linearly and shows no anomaly [14,16]. The same tendency is reproduced by computer simulations for concentrations lower than 0.70 .

\subsection{Temperature-dependent magnetic susceptibility}

Apart from the experimentally measured specific heat and magnetization, we also determined the diagonal components of the magnetic susceptibility tensor, $\chi$, for samples with 

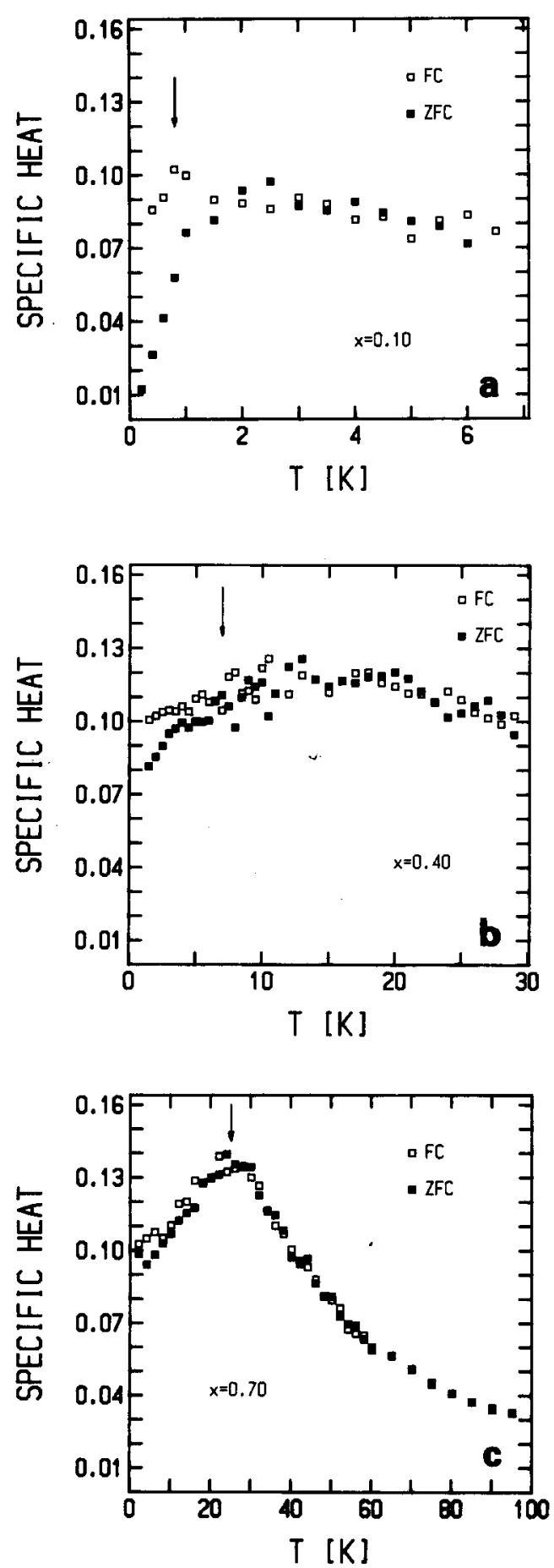

Fig. 5. Specific heat as a function of temperature for various concentrations of spins, $x$. (a) $x=0.10$, (b) $x=0.40$, (c) $x=$ 0.70. The arrows indicate critical temperatures obtained from the magnetization for corresponding $\boldsymbol{x}$.

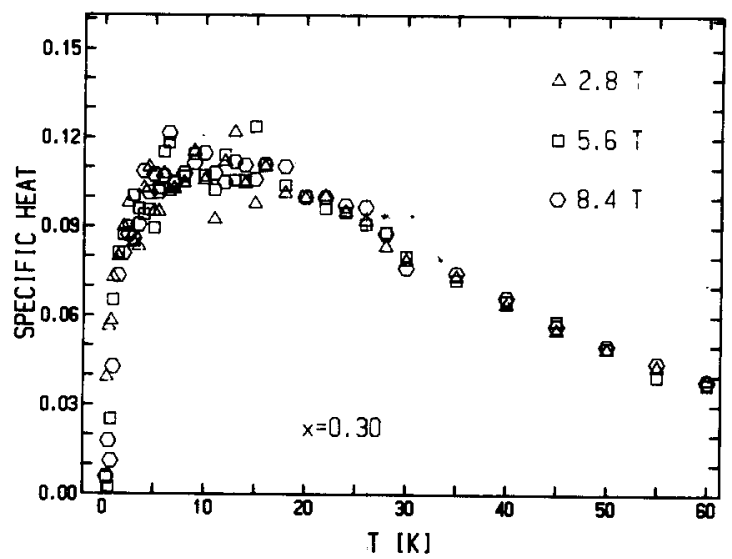

Fig. 6. Specific heat versus temperature for different external magnetic fields $B=2.8,5.6,8.4 \mathrm{~T}$. Spin concentration, $x=0.30$.

different concentrations. For systems below 0.30 we found that $x x, y y$ components of $\chi$ almost do not depend on temperature, while $\chi_{z z}$ goes down with temperature. We present these results in fig. 7. The low values of $\chi_{2 z}$ results from the fact that the susceptibility tensor was determined for high external magnetic field in the $z$-direction. However, for higher concentrations, this behaviour was not observed and there was no distinction between diagonal components of $x$. Such anisotropy of magnetic susceptibility, for samples with small values of $x$, has not been observed experimentally.

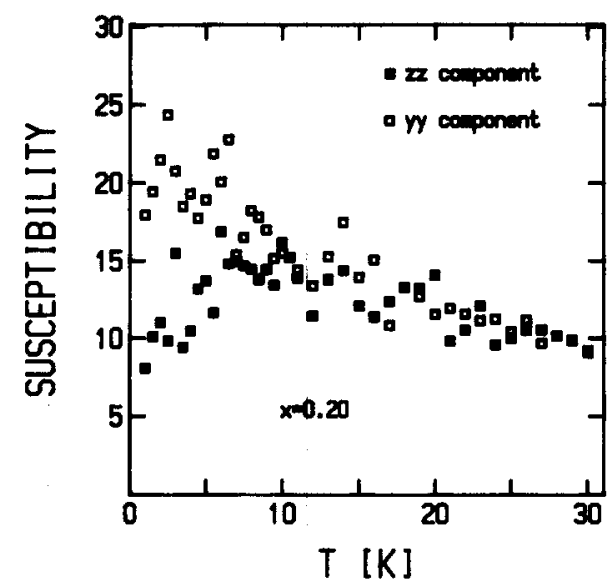

Fig. 7. Magnetic susceptibility as a function of temperature for low concentration of spins, $x=0.20$. The difference between $y y$ component (open squares) and $z 2$ (closed squares) is seen. 

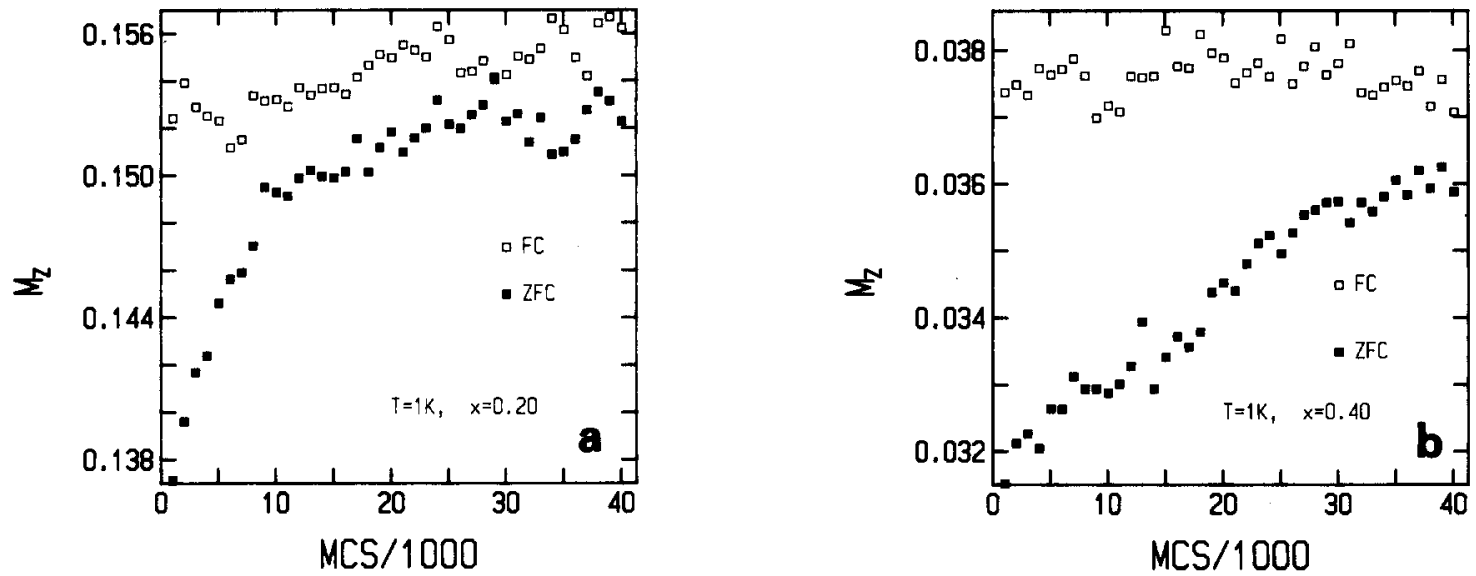

Fig. 8. Magnetization as a function of Monte Carlo steps (MCS). (a) Number of spins, $N_{\mathrm{s}}=2195$, concentration of spins, $x=0.20$; (b) $N_{\mathrm{s}}=7861, x=0.40$.

\subsection{Dependence of magnetization and energy on number of Monte Carlo steps}

For typical simulations which allowed us to get the temperature dependence of thermodynamical quantities, we used about 2000 MCS for each temperature point. Additionally, we did longer computations of 40000 MCS for several temperatures and concentrations of spins.

From these long runs we wanted to observe the large "time"-scale evolution of magnetization and energy. Another point was to determine the number of MCS's necessary for the system to reach thermodynamical equilibrium and the corresponding number of MC steps needed to average physical quantities. We found that for the same temperature and concentration of spins, the ZFC and FC systems behaved in different ways below the critical temperature.

In general, rejecting several hundreds of MCS's and taking 1000 steps for the average $F C$ values was enough, but in the ZFC case the magnetiza-
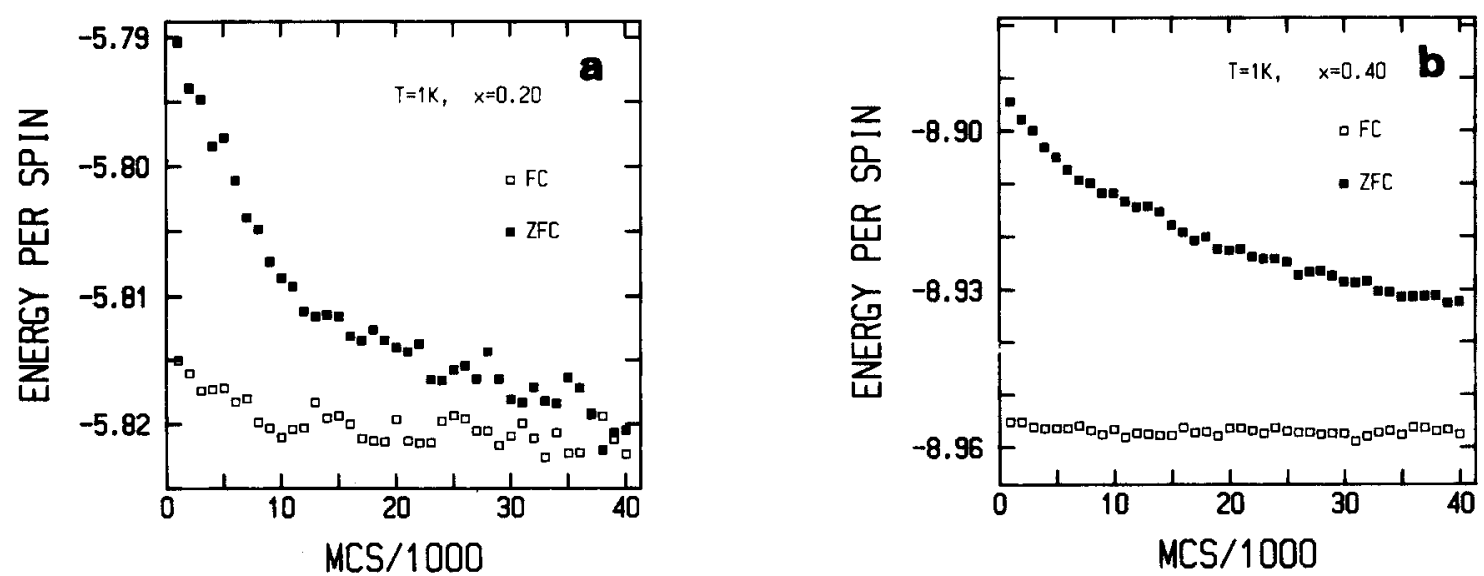

Fig. 9. Energy per spin as a function of Monte Carlo steps (MCS). (a) Number of spins, $N_{s}=2195$, concentration of spins, $x=0.20$; (b) $N_{s}=7861, x=0.40$ 
tion increases (energy decreases) steadily over tens of thousands of MCS's. Using the FC "time"-scale for ZFC system produced the results presented in sections 3.1 and 3.2. The examples of long "time" evolution of magnetization and energy are given in figs. 8 and 9.

In fig. $8 \mathrm{a}$ we plot the magnetization as a function of Monte Carlo steps for $T=1 \mathrm{~K}, x=0.20$ and $N_{\mathrm{s}}=2195$. Each point on the curve represents average values of $M_{z}$ over 1000 configurations. The ZFC magnetization tends toward the FC which is almost constant with MCS. In fig. $8 \mathrm{~b}$ the same results are presented for $x=0.40$ and $N_{\mathrm{s}}=$ 7861. It can be seen from both plots that for lower concentration of spins less MCS's are needed, for the ZFC $M_{z}$ to reach a value corresponding to the FC level. In fig. 9a, b energies are plotted for the same values of $T, x$ and $N_{\mathrm{s}}$. Again the FC curves are almost constant and ZFC values tend toward them.

We also did longer simulations for $T=3,10 \mathrm{~K}$. For samples with $x=0.20$ and $T_{\max } \approx 2 \mathrm{~K}$ we found no differences in behaviour of ZFC and FC quantities. However, systems with $x=0.40$ and $T_{\max } \approx 7 \mathrm{~K}$ behaved in the same way only for $T=10 \mathrm{~K}$, but at $3 \mathrm{~K}$ the $\mathrm{ZFC}$ energy and magnetization were approaching $\mathrm{FC}$ values.

\subsection{Other effects}

From our numerical work we could produce results which are in good agreement with experiment. However, by changing simulation parameters we also obtained another picture, which is not experimentally observed.

In fig. 10 we present $M_{z}$ curves for ZFC \& FC systems for $x=0.30$. The size of the sample was 1200 spins. In this simulation temperature was changed in greater steps than usual. In an experiment, these changes correspond to a greater rate of cooling or heating of a real sample. Comparing fig. 10 to fig. 1a we can see that the maximum for the ZFC magnetization is shifted to the right and the FC curve is shifted to the left. Such effects were neglected in the experimental works. Some evidence that empirical results depend on rate of cooling was reported in ref. [17].

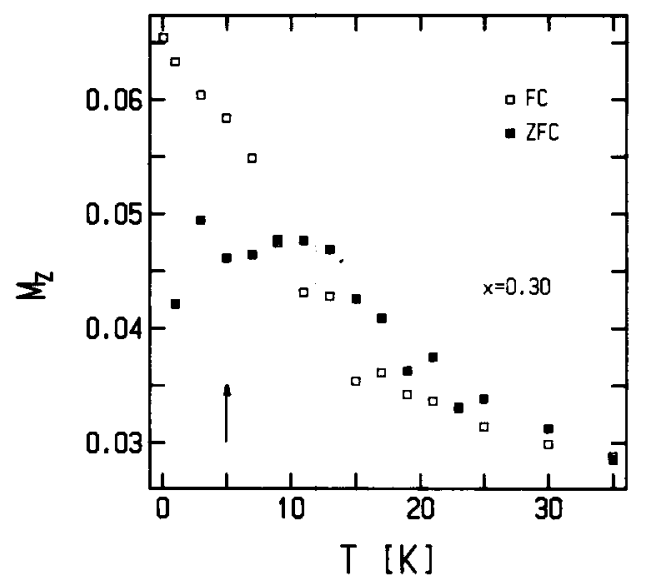

Fig. 10. Temperature dependence of the magnetization in case of rapid temperature changes, (8 "measurements" in 1-15 K interval), $N_{\mathrm{s}}=1200, x=0.30$. The arrow indicates the critical temperature for magnetization of a typical $x=0.30$ run [see fig. la for comparison].

Doing several ZFC landings in high concentrations $(x=0.70,0.80)$, we found that starting points of ZFC curves for magnetization are distributed randomly over the area where the FC curve goes through. This was the reason that not only one curve for the ZFC system was observed in our experiments but a whole family of curves. It was even possible to get to a situation where the behaviour of $\mathrm{ZFC}$ and FC systems was reversed. This is probably a numerical artifact coming from high fluctuations of both magnetization curves, which are very flat and close each to other. For lower concentration of spins, the difference between ZFC and FC magnetizations was greater and ZFC landings were far from FC curves.

\section{Final conclusions}

\subsection{Magnetic phase diagram}

Using the Monte Carlo simulations described we were able to reproduce numerically the experimental magnetic phase diagram, extending it to the physically inaccesible concentrations of $\mathrm{Mn}$ ions in $\mathrm{Cd}_{1-x} \mathrm{Mn}_{x} \mathrm{Te}$. Since the critical temperature for every concentration is scaled in our calculations by $J_{n n}$, fitting the numerical results for the 
phase diagram to experimental data allows us to derive a value of $J_{n n}$ and to study its possible dependence on concentration. The same method can be used for other SMSC with unknown $J_{\mathrm{nn}}$.

The common feature of the model system over the whole range $0.05 \leq x \leq 0.90$ was the difference of the ZFC and FC magnetization, energy and specific heat. However, more detailed studies have allowed us to distinguish three different concentration-dependent regions.

In the near percolation region with low concentration, $0.05 \leq x \leq 0.20$, we observed spin glass behaviour with a cusp in ZFC magnetization and a broad maximum of specific heat shifted to higher temperature. The dominant features were the anisotropy of the magnetic susceptibility and smooth curves for the magnetization. The relaxation "times", measured in MCS's, for ZFC energy and magnetization, were short; the system quickly evolved to FC state.

In the intermediate region, $0.30 \leq x \leq 0.60$, the system also behaved like a spin glass, but with a longer relaxation of the ZFC quantities. The magnetization curves showed larger fluctuations and the difference between perpendicular and parallel field components of the magnetic susceptibility vanished. As in the first region, the difference between the cusp in ZFC magnetization and the broad maximum of the specific heat was distinct.

The last interval of concentrations, $0.70 \leq x \leq$ 0.90 , is partially not accessible in experiment, since $\mathrm{Cd}_{1-x} \mathrm{Mn}_{x}$ Te crystallizes in fcc structure only for $x \leq 0.70$ [2]. The main feature of this area in our simulation was the common position of magnetization and specific heat maxima. This result is typical for an antiferromagnetic phase, which is also apparently evidenced in experiment $[10,18]$. In this region we observed large fluctuations of physical quantities and long relaxations of the ZFC magnetization and energy toward FC values. The $M_{z}$ curves for the ZFC and FC systems were very flat and close each to other.

\subsection{New results}

Apart from the results which were compared with experiment we also observed some new effects not previously reported. Anisotropy of the magnetic susceptibility for low concentration of spins is one such example. Also the different temperature-dependence for the ZFC and the FC specific heat has not been observed experimentally.

Investigation of the energy and magnetization during long runs of 40000 MCS's gives some insight in the time-dependent behaviour of the ZFC states, under the assumption that Monte Carlo steps are related in some way to real time in an experiment. If this condition is fulfilled, we can derive the conclusion that the ZFC system is a metastable state relaxing toward the FC one. The time of relaxation depends on the concentration of spins - the higher value of $x$, the greater time.

Another effect, which can be attributed to time scales, is the dependence of the magnetization results on the rate of cooling. Quick changes in temperature shift the position of the cusp for the ZFC magnetization. This effect can explain the different critical temperatures reported by various authors for the samples with the same concentrations of $\mathrm{Mn}$ ions [10-13].

In summary, we conclude that the Monte Carlo method is a useful tool to investigate SMSC systems and produces results in good agreement with experiment as well as predicting some new effects, not observed experimentally at present.

\section{Acknowledgements}

We acknowledge that most of the computations presented in that paper were done at the University of Regensburg. We would like to express our sincere thanks to Professor Wolfgang Gebhardt and his group at the Department of Physics for the hospitality they extended to one of us (JM) during the stay at the Regensburg University.

\section{References}

[1] R.R Galazzka and J. Kossut in: Landolt-Bornstein, New Series $17 b$, eds. O. Madelung, $M$. Schultz and $M$. Weiss (Springer, Berlin, 1982) p. 302.

[2] J. Bąk, U. Dębska, R.R. Galązka, G. Jasiolek, E. Mizera and B. Bryza, Suppl. Acta Crystallogr. A 34 (1978) 245.

[3] W.H. Brumage, C.R. Yager and C.C. Lin, Phys. Rev. 133 (1964) A765. 
[4] T.M. Giebultowicz, J.J. Rhyne, W.Y. Ching, D.L. Huber and R.R. Galazkka, J. Magn. Magn. Mat. 54-57 (1986) 1149.

[5] B.E. Larson, K.C. Hass and L. Aggarwal, Phys. Rev. B 33 (1986) 1789.

[6] A. Lewicki, J. Spalek, J.K. Furdyna and R.R. Galazka, Phys. Rev. B 37 (1988) 1860.

[7] K. Binder and A.P. Young, Rev. Mod. Phys. 58 (1986) 801.

[8] H. Kett, W. Gebhardt and U. Krey, J. Magn. Magn. Mat. 46 (1984) 5.

[9] Monte Carlo Methods in Statistical Physics, ed. K. Binder, vol. 7 (Springer, Berlin, 1979).

[10] R.R. Galazzka, S. Nagata and P.H. Keesom, Phys. Rev. B 22 (1980) 3344.

[11] M.A. Nowak, O.G. Symko, D.J. Zheng and S. Oseroff, J. Appl. Phys. 57 (1985) 3418.
[12] M. Escorne, A. Mauger, R. Triboulet and J.L. Tholence, Physica B 107 (1981) 309.

[13] S.B. Oseroff, Phys. Rev. B 25 (1982) 6584.

[14] A. Twardowski, C.J.M. Denissen, W.J.M. de Jonge, A.T.A.M. de Waele, M. Demianiuk and R. Triboulet, Solid State Commun. 59 (1986) 199.

[15] J. Spalek, A. Lewicki, Z. Tamawski, J.K Furdyna, R.R. Galazka and Z. Obuszko, Phys. Rev. B 33 (1986) 3407.

[16] S. Nagata, R.R. Galazka, G.D. Khattak, C.D. Amarasekara, J.K. Furdyna and P.H. Keesom, Physica B 107 (1981) 311.

[17] R.R. Galazka, W.J.M. de Jonge, A.T.A.M. de Waele and J. Zeegers, Solid State Commun. 68 (1988) 1047.

[18] T.M. Giebultowicz, J.J. Rhyne, W.Y. Ching, D.L. Huber, J.K. Furdyna, B. Lebech and R.R. Galgzka, Phys. Rev. B 39 (1989) 6857. 